\title{
Ganglioglioma: Unusual Ultrastructural Features of Neuronal Cells.
}

\author{
K.L. Ho
}

Department of Pathology. Henry Ford Hospital. Detroit, MI 48202

Ganglioglioma is uncommon, slow growing neoplasm of the central nervous system, generally found in the temporal lobe of young individuals with a clinical history of seizures. The tumor is a mixed tumor composed of large, variably dysmorphic neurons and a glial component, mostly astrocystic in nature. Although the clinicopathological correlation of this tumor iswell documented, few detailed ultrastructual studies have been reported ${ }^{[1,2,3]}$.

This report described several unusual ultrastructural features of the neuronal cells in five cases of ganglioglioma: (1) Perikaryonal aggregates of distended endoplasmic reiculum, neurofilaments and dense core granules. Most endoplasmic reticulum contained granulofibrillary material surrounded by an electron-luccent zone (FIG.1). (2) Aggregates of synaptic vesicles, dense-core granules and various sized electron-dense bodies, some composed entirely of concentrically laminated membranes in distended neurites. (3) Abnormal accumulation of neurofilaments, some with central core of mixed microtubules, vesicles and dense bodies is distended neurites. (4) Hirano bodies (eosinophilic rod-like structures), highly organized crystalloid arrays of interlocking filaments that display lattice like configuration. Homogenous electron-dense material may appear as patches permeating the fibrillar structure (Fig.2). (5) Lafora bodies (polyglucosan bodies), round to oval non-membrane bound inclusion composed of short branching filaments about 7-10 nm in diameter and granular structures (Fig. 3). (6) Pick's bodies, perikaryonal aggregates of straight neurofilaments with or without ribosome association often mixed with vesicles, myelin figures and dense bodies. (7) Curvilinear membranous formations (curvilinear bodies) as seen in ceroid lipofuscinosis. (8) Tubular inclusion, aggregates of smooth-membrane bound ramifying tubules of 80-100 nm in diameter (Fig.4). (9) Aggregates of vacuoles, myelin figures and concentric or lamellar membranes in enlarged neurites.

The present findings suggest that the intracellular alterations of neuronal cells are common in gangliogliomas. Similar alterations have been observed in other neoplasms such as gangliocytoma, ganglioneuroma and ganglioneuroblastoma, and in some degenerative diseases of neurons ${ }^{[4,5]}$. The significance of these intracellular alterations in relation to any metabolic change postulated to occur in neoplastic neuronal cells is nuclear. The detailed morphological descriptions of the ultrastructural alterations may be important in seeking the mechanism of neuronal degenerative disease of the central nervous system.

\section{References}

[1] DC Miller, FF Lang, FJ Epstein et al., J Neuro Surg 79 (1993) 859.

[2] HGJ Krouwer, RL Davis, MW McDermott et al., J Neuro Oncol 17 (1993) 139.

[3] T Hirose, BW Scheithauer, MBS Lopes et al., Cancer 79 (1997) 989.

[4] A Hirano. Textbook of Neuropathology. Williams and Wilkins. Baltimore (1991) p 1-94.

[5] RE McLendon, DS Enterline, RD Tien et al., Russel and Rubinstein's Pathology of Tumors of the Nervous System. 6th ed. Arnold: London (1998) pp 307-571. 


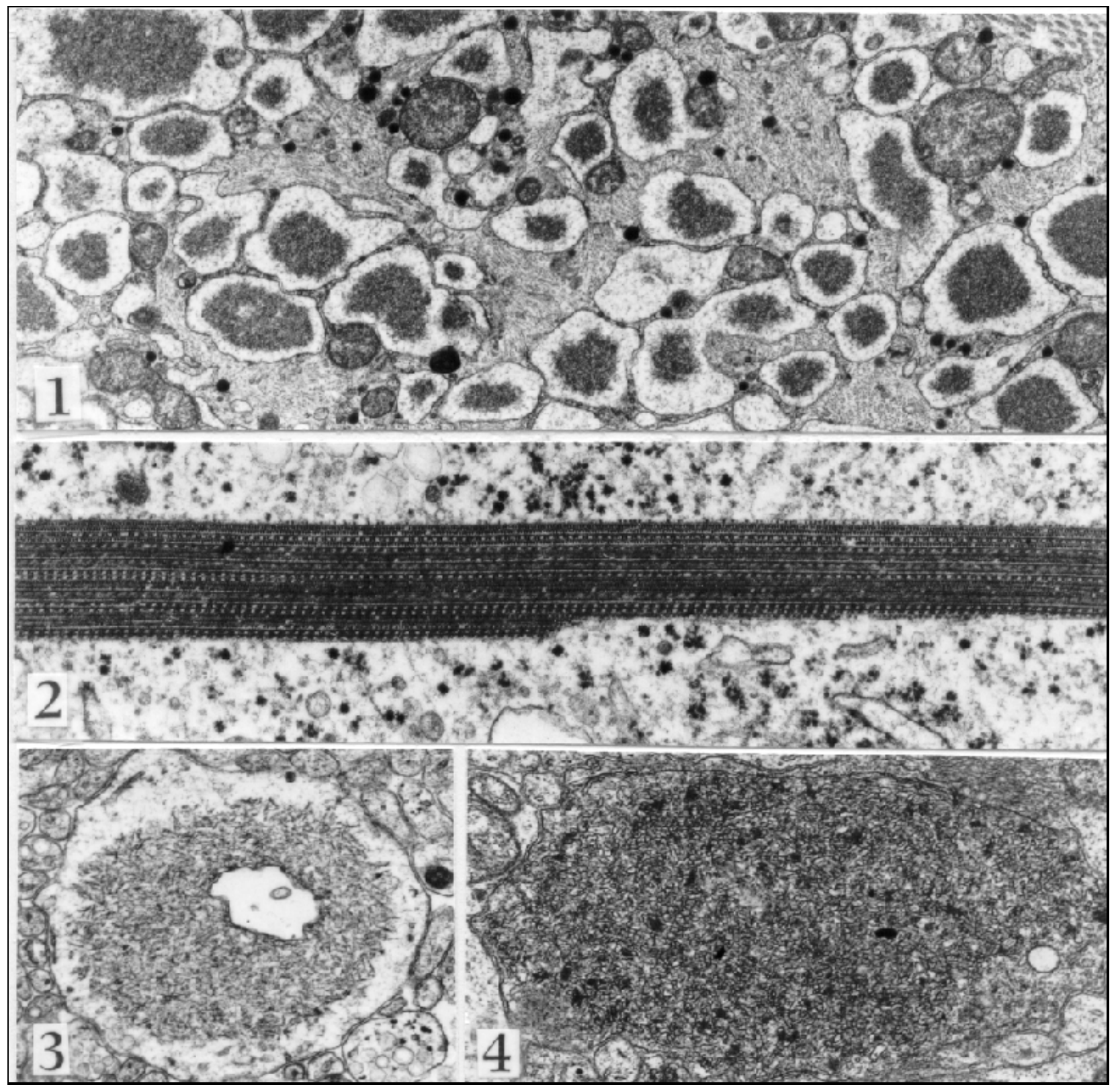

FIG. 1. Perikaryonal aggregate of neurofilaments, dense core granules and dilated endoplasmic reticulum with central granulofibrillary inclusions. $x$ 15,000.

FIG. 2. Elongated Hirano body in the Perikaryon. x 32,000

FIG. 3. Lafora body (polyglucosan body) in enlarged neurite. $x 22,800$

FIG. 4. Aggregate of tubular structure in distended neurite. x 21,500 